\title{
Efficacy of $1.23 \%$ acidulated phosphate fluoride gel on non-cavitated enamel lesions: a randomized clinical trial
}

\author{
Gabriela Maltz GOLDENFUM(a) iD \\ Natália Caldeira SILVA(a) \\ Ingrid dos Anjos ALMEIDA(a) iD \\ Matheus NEVES(b) \\ Berenice Barbachan e SILVA ${ }^{(b)}$ iD \\ Juliana Jobim JARDIM(b) \\ Jonas Almeida RODRIGUES(a) \\ (a) Universidade Federal do Rio Grande do Sul \\ - UFRGS, School of Dentistry, Department \\ of Surgery and Orthopedics, Porto Alegre, \\ RS, Brazil. \\ (b) Universidade Federal do Rio Grande do Sul \\ - UFRGS, School of Dentistry, Department \\ of Preventive and Social Dentistry, Porto \\ Alegre, RS, Brazil.
}

Declaration of Interests: The authors certify that they have no commercial or associative interest that represents a conflict of interest in connection with the manuscript.

Corresponding Author:

Jonas Almeida Rodrigues

E-mail: jorodrigues@ufrgs.br

Submitted: April 29, 2020

Accepted for publication: October 22, 2020

Last revision: December 8, 2020
Abstract: A randomized controlled parallel clinical trial was conducted to evaluate the efficacy of the association of $1.23 \%$ topical professional acidulated phosphate fluoride (APF) gel with oral hygiene and dietary instructions on the arrest of active non-cavitated lesions in permanent, mixed, and temporary dentition in children between 3 and 12 years of age. Ninety-eight caries-active children were randomly divided into two groups: a fluoride gel group $(n=49)$ and a non-fluoridated gel group ( $n=49$ ). Each group received up to eight weekly applications of gel, weekly professional toothbrushing with oral hygiene instructions and dietary counselling. Caries lesions were assessed qualitatively through visual-tactile criteria performed in three stages: initial, intermediate, and final. Regression models were applied to identify risk indicators for caries control. Between-group comparison regarding the time taken to arrest the lesions was performed using Chi-squared and Fisher's exact tests. Seventeen children did not receive the allocated intervention, and one was lost to follow-up ( $n=80)$. There was no difference between the control and placebo groups regarding the time to the arrest of the lesion ( $p$ >.05). The treatments showed similar results without significant statistical difference ( $p=0.33$; 95\%CI: 0.32-1.48). No adverse effects were observed. It can be concluded that no additional effect of the association of $1.23 \%$ APF gel with oral hygiene using fluoride dentifrice and dietary instructions on the arrest of active non-cavitated lesions could be established. We can also confirm the importance of toothbrushing frequency and, consequently, the visible plaque reduction in the control of caries activity. RBR-37V5S3

Keywords: Dental Caries; Acidulated Phosphate Fluoride; Pediatric Dentistry.

\section{Introduction}

Studies still show a considerable number of children presenting with dental caries worldwide. The level of dental caries continues to be high in low-income and low educational-level populations. ${ }^{1,2}$ The disease consists of a dynamic de- and remineralization process resulting in a loss of minerals over time, varying clinically from a white spot to a cavity. Incipient carious lesions are significantly more prevalent than cavitated lesions, ${ }^{3,4}$ and the presence of initial lesions in deciduous 
dentition is a predictor of an increase in caries in permanent dentition. ${ }^{5}$

Understanding the dynamic nature of the caries process, in which progression can be disrupted at any stage, increases the importance of clinically assessing the status of the lesion. ${ }^{6}$ The possibility of stopping the progression of enamel lesions through non-invasive treatments has encouraged professionals to better understand the disease process. The arrest of an active non-cavitated lesion is fundamentally related to the triad of oral hygiene, diet control, and fluoride.

Since the main mechanism of action of dental caries occurs at the tooth-plaque interface, several topical clinical methods have been developed. Fluoride has emerged as a conservative therapeutic option to decrease the speed of tooth decay, restricting demineralization, and stimulating remineralization. Among the fluoride-containing products, topical professional acidulated phosphate fluoride (APF) is frequently used. Some authors have recommended topical methods with higher concentrations and higher frequency of use, specifically four to eight weekly or fortnightly sessions for conservative treatment. ${ }^{7}$

In vitro ${ }^{8}$ and in situ ${ }^{9}$ studies have confirmed the action of APF gel, showing its remineralizing ability in re-hardening enamel, even when used in conjunction with fluoride dentifrice, showing an additional effect. However, only two double-blind randomized controlled school-based trials in permanent dentition with schoolchildren aged 7 to 12 years have been performed. These studies converged to the same results: no additional benefits were found after treating incipient carious lesions with fluoride gel when fluoride dentifrice was also being used, considering that the arrest of the lesion was the main outcome. ${ }^{10,11}$ Nevertheless, no reports are available to determine the frequency and duration of application for high fluoride concentration products in the remineralization of early enamel caries lesions in a clinical setting. Further research on non-surgical treatment of incipient carious lesions is warranted. ${ }^{12}$

Therefore, the aim of this randomized controlled clinical trial was to evaluate the efficacy of $1.23 \%$ APF gel with oral hygiene (toothbrushing with fluoride dentifrice and flossing) and dietary instructions on the arrest of active non-cavitated lesions in permanent, mixed, and temporary dentition in children aged between 3 and 12 years. The null hypothesis was that there is no additional effect of the association of $1.23 \%$ APF gel with oral hygiene using fluoride dentifrice and dietary instructions on the arrest of active non-cavitated lesions.

\section{Methodology}

\section{Study design and sampling procedure}

This randomized controlled parallel clinical trial was conducted between 2017 and 2018 on 98 children between 3 and 12 years of age who sought treatment at the Pediatric Clinic at the Dental Teaching Hospital at the Federal University of Rio Grande do Sul, Porto Alegre, southern Brazil. Children and their legal guardians signed a written informed consent form (Ethics Committee Approval 2.224.755 and Brazilian Clinical Trials Registry RBR-37V5S3).

A sample size calculation was performed assuming a difference between the test and control groups of $20 \%$ at $\alpha=5 \%$ with a power of $80 \%$. The number of required treatments per group was 38 . Considering a dropout rate of $25 \%$, a total sample of at least 47 patients per group was set.

To be included in the study, children had to present at least one initial enamel lesion in the smooth or occlusal surface in deciduous or permanent dentition, have access to fluoridated water $(0.7 \mathrm{ppmF})$, and use standard fluoride dentifrice ( $>1,000 \mathrm{ppmF})$. Children with systemic alterations that could interfere with the development of caries, cognitive impairment preventing adherence to treatment, and topical fluoride applications received in the last six months before the beginning of the study were not included. Children who did not attend all dental visits were excluded from the study.

\section{Training and calibration}

Clinical examinations and treatments were performed by two properly trained examiners (GMG and NCS). The training for the clinical examination was done through photographs of caries lesions in various stages with discussion of doubtful points until consensus was reached. The calibration of the 
visual-tactile clinical examination was performed through the examination of 15 subjects at weekly intervals, supervised by an experienced calibration examiner (gold standard, JAR). The intra- and inter-examiner reproducibility was calculated using the Cohen kappa coefficient, with the minimum value required being 0.70 . The kappa inter-examiner value was 0.78 , and the intra-examiner values were 0.80 (GMG) and 0.78 (NCS).

\section{Study groups, randomization, and blinding}

The subjects were randomly assigned to either the test or control group. The test group (fluoride gel: FG) included caries control through a $1.23 \%$ APF gel, oral hygiene (toothbrushing with fluoride dentifrice and flossing), and dietary counselling. The control group (non-fluoridated gel: NFG) included caries control through placebo gel, oral hygiene (toothbrushing with fluoride dentifrice and flossing), and dietary counselling.

The randomized sequence was generated through a website (randomization.com). Allocation concealment was secured by keeping the randomization sequence in separate sequentially numbered dark envelopes. A person other than the operators performed these procedures. The envelopes had a carbon paper, and the examiner and legal guardian of the participant signed and dated the outer surface of the envelope just before opening it.

The operators, assistants, and participants were blinded to the groups to which they were allocated. To ensure the blindness of the research team and participants, the gels (test and placebo) presented the same color, taste, smell, consistency, and appearance, and were kept in similar bottles with similar labels. A person other than the operators prepared the gel tubes and provided them right after the randomization procedure. The statistician was also blinded to the treatment groups.

\section{Clinical exams and interventions}

The two previously trained dentists performed clinical examinations at the Pediatric Clinic at the Dental Teaching Hospital. Oral hygiene was assessed by recording the visible plaque index $(\mathrm{VPI})^{13}$ and gingival blood index (GBI). ${ }^{13}$ Prophylaxis was performed with dental floss, a rubber cup on smooth surfaces, a Robson brush on occlusal surfaces, and dentifrice at 1,100 ppmF. Lesions were detected by visual-tactile examination with a clean, dry, and illuminated surface under cotton-roll isolation. Visual examination of the caries lesions was conducted after tooth cleaning. Dental caries was recorded based on two indices of evaluation of caries extent/severity and activity: The International Caries Detection and Assessment System ${ }^{14}$ complemented by the Nyvad Criteria. ${ }^{15}$ Topical gel application (TGA) was performed in both the upper and lower jaws after prophylaxis, along with brushing for 1 minute with a toothbrush, using a suction tube (protocol adapted from de Oliveira et $\mathrm{al}^{16}$ ). In the FG group, TGA was performed with 1.23\% APF gel (DFL, Sultan Topex, Rio de Janeiro, Brazil). In the NFG group, TGA was performed with a placebo gel (same color, taste, smell, consistency, and appearance but without fluoride; DFL, Sultan Topex). Toothbrushes (Colgate/Palmolive, São Bernardo do Campo, Brazil) and fluoride dentifrice (1,100 ppmF, Colgate/Palmolive) were provided to the children to be used at home throughout the trial. Both groups had weekly professional toothbrushing with oral hygiene instructions and weekly dietary counselling. Parents of children under 6 years of age were instructed to use a pea-sized amount of fluoride dentifrice. Children over 6 or those who could spit out the dentifrice themselves were instructed to brush their teeth themselves using a small amount of fluoride dentifrice.

Patients were followed up once a week for 13 weeks. In the first week, the initial examination was conducted, a questionnaire for personal data collection was answered by the legal guardian, followed by a 24-h dietary diary to assess the children's diet, and the first TGA was completed. Both groups received the same procedures weekly (prophylaxis, exams, TGA, oral hygiene instructions, and dietary counselling) for up to 8 weeks. There was no minimum TGA number, which could be from one to eight applications, until reaching arrest. After the weekly clinical examination, if the patient presented clinical arrest of the lesions, from that moment onward, he or she would no longer come to the other visits, being recalled only at weeks 9 and 13. In week 9, an intermediate examination 
was performed, and a new 24-h dietary diary was provided. Three months after the start of the study (week 13), a final examination was performed. The possible adverse effects were collected and recorded in a specific form.

\section{Statistical analysis}

The independent variables were initially described using absolute and relative frequencies. Multivariable analysis was used to evaluate the association between the variables and the outcome (carious lesion control) with adjustment for confounding factors for the independent variables. A Poisson regression model with robust variance was used to determine the crude and adjusted prevalence ratios and their respective $95 \%$ confidence intervals (CIs) at a level of significance of $5 \%$. Crude analyses were initially performed. Variables with $\mathrm{p}<0.05$ were considered to be associated with the outcome. The statistical significance of the prevalence ratio was evaluated using the Wald test. The Hosmer-Lemeshow test was used to verify the model adjustment. The comparison between baseline characteristics in both groups was performed using the chi-squared and Fisher's exact tests. To compare the mean values of VPI in the initial and intermediate exams, the t-test was used. The statistical package used was SPSS Statistics (v.21.0, IBM; Armonk, USA). Sample power was calculated using the "pwr".

$\mathrm{R}$ software package, version 3.5.1 (R Foundation, Vienna, Austria) and a significance level of $5 \%$ was used.

\section{Results}

Ninety-eight patients were initially included in the study. Eighty patients completed the study protocol. The power of the sample test was $87.86 \%$. No adverse effects were observed. Figure shows the CONSORT flow diagram of the clinical trial. The average age of the sample was $7.53 \pm 2.28$ years. There were 41 females (51.2\%) and 39 males (48.8\%). The most frequently reported education level of mothers was high school (57.5\%). There were no significant differences in baseline characteristics between groups (Table 1).

Although treatments showed similar results without significant statistical difference, in the FG group, $74.3 \%$ of children had a total arrest of the active non-cavitated lesions, and in the NFG group, $78 \%$ of children had a total arrest of the active non-cavitated lesions. The mean number of APF applications until the total arrest of lesions was $5.28 \pm 2.28$ in both groups. Overall, 438 active non-cavitated lesions were treated. There were 7 lesions that ended up cavitated (1.5\%), 47 remained active, and 384 became inactive non-cavitated lesions. Out of the 438 initial lesions, 374 were in smooth surfaces, and 64 were in occlusal surfaces. Of the 47 lesions that remained active after treatment, 33 were in permanent teeth, and most of them were on smooth surfaces.

Table 2 presents the results of the simple and multiple regression analyses. The treatments showed similar results $(p=0.33)$. The only significant variable associated with caries lesion control was toothbrushing frequency at least three times per day $(\mathrm{OR}=0.31$, $p=0.04$ ). There was a significant statistical difference when the means of the initial and intermediate VPI were compared $(p=0.02)$.

\section{Discussion}

This study is the first clinical trial to evaluate the efficacy of $1.23 \%$ APF gel in permanent, mixed, and temporary dentition. The association between APF gel, biofilm control using fluoride dentifrice, and dietary counselling to control incipient carious lesions showed no additional effect of fluoride gel.

The only significant variable associated with caries lesion control was toothbrushing frequency using fluoride dentifrice. Children who reported brushing their teeth three times or more per day had a $70 \%$ greater chance of the arrest of lesions. They also had greater access to fluoride through regular brushing with fluoride dentifrice. Few epidemiological studies have shown an association between toothbrushing frequency and caries. Researchers cannot isolate the effect of toothbrushing because it is always associated with the use of fluoride. A systematic review and meta-analysis that evaluated the effect of toothbrushing frequency on the incidence and increment of carious lesions concluded that individuals who brush their teeth infrequently are at greater risk for the incidence or increment of new carious lesions 


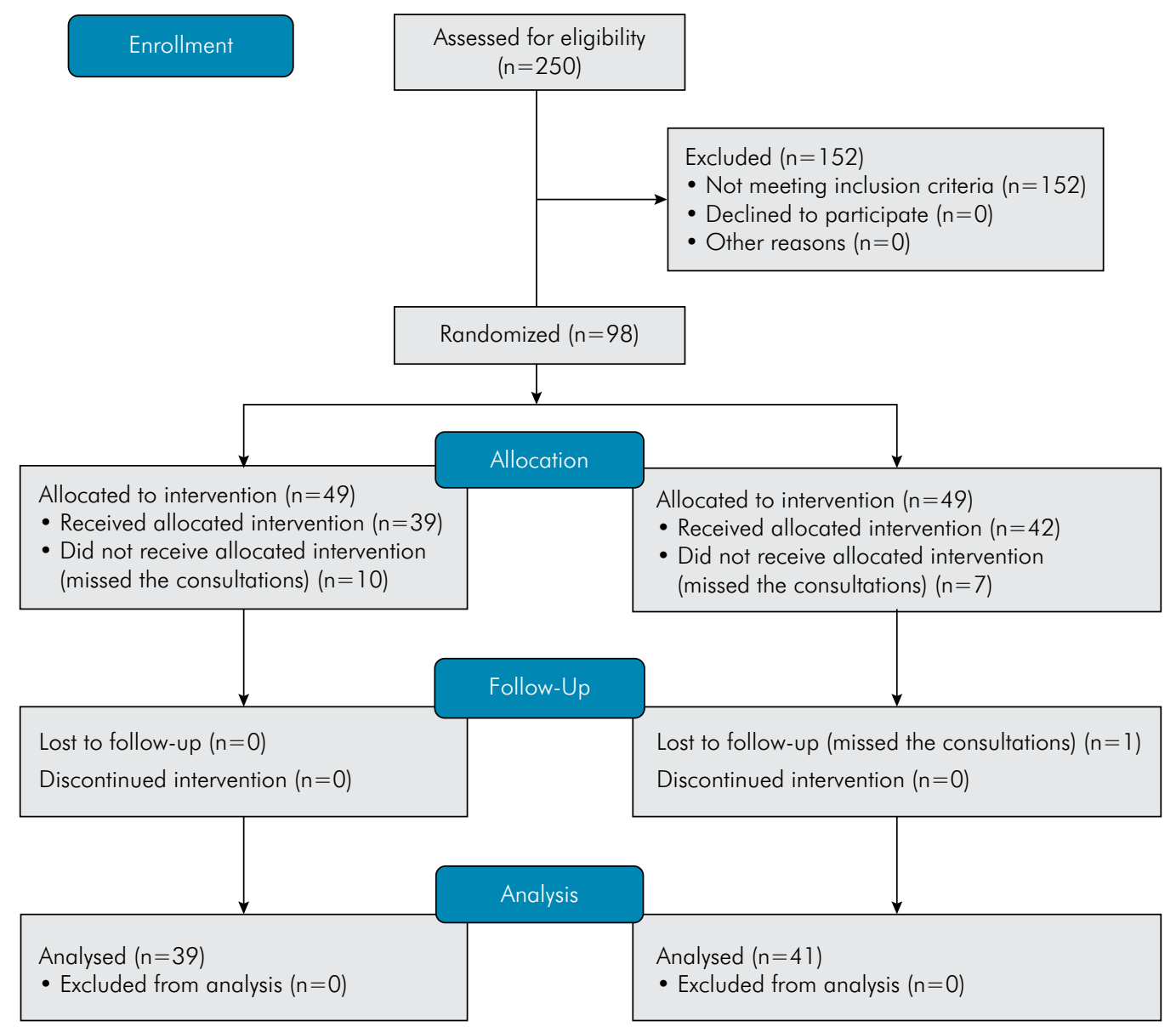

Figure. Consort flow diagram.

than those brushing more frequently. ${ }^{17}$ Treasure et al. ${ }^{18}$ also showed that toothbrushing had a positive association with the number of sound and unrestored teeth in the adult population.

Although the percentage of arrestment was numerically lower in the FG group, no statistical difference was found between the treatments. As there was no difference between the fluoride gel and placebo gel group, the reason for the arrest was probably the weekly professional prophylaxis and regular biofilm removal using the toothbrush and fluoride dentifrice. The mechanical removal of bacterial plaque performed by the examiners was the only variable that could be controlled. There was a significant statistical difference when the t-test was performed to compare the means of initial and intermediate VPI, showing that children controlled their active caries status and reduced bacterial plaque.
The attrition of the porous surface in the lesion area indicates that functional wear and toothbrushing were responsible for the arrest through the disturbance and removal of bacterial deposits. ${ }^{19}$ Surface abrasion by daily brushing with some redeposition of minerals is believed to be responsible for the regression of the lesions. It is known that toothbrushing with fluoridated dentifrices alone can lead to the arrest of white spot lesions. ${ }^{20}$

The frequency of sugar intake did not decrease, showing that changing dietary habits in children may be a challenge in a clinical setting. Studies report that the 24-h dietary diary method tends to underestimate an individual's diet when applied in a single opportunity, ${ }^{21}$ primarily because it is a self-administered food consumption frequency questionnaire filled in by the parents. A systematic review has highlighted the diversity of reporting 
Table 1. Baseline characteristics by treatment group.

\begin{tabular}{|c|c|c|c|c|c|c|c|}
\hline \multirow{2}{*}{ Variable } & \multicolumn{2}{|c|}{ Fluoride gel group (FG) } & \multicolumn{2}{|c|}{$\begin{array}{l}\text { Non-fluoride gel group } \\
\text { (NFG) }\end{array}$} & \multicolumn{2}{|c|}{ Total } & \multirow{2}{*}{$\mathrm{p}$-value } \\
\hline & $\mathrm{n}$ & $\%$ & $n$ & $\%$ & $\mathrm{n}$ & $\%$ & \\
\hline \multicolumn{8}{|l|}{ Age (years) } \\
\hline $3-8$ & 25 & 64.1 & 25 & 61.0 & 50 & 62.5 & $0.063^{\circ}$ \\
\hline $9-12$ & 14 & 35.9 & 16 & 39.0 & 30 & 37.5 & \\
\hline \multicolumn{8}{|l|}{ Gender } \\
\hline Female & 20 & 51.3 & 21 & 51.2 & 41 & 51.2 & $0.634^{\circ}$ \\
\hline Male & 19 & 48.7 & 20 & 48.8 & 39 & 48.8 & \\
\hline Mother's educational level & & & & & & & $0.712^{b}$ \\
\hline Elementary school & 8 & 20.6 & 12 & 29.3 & 20 & 25.0 & \\
\hline High school & 25 & 64.0 & 21 & 51.2 & 46 & 57.5 & \\
\hline Higher education & 6 & 15.4 & 8 & 19.5 & 14 & 17.5 & \\
\hline DMFT & & & & & & & $0.628^{b}$ \\
\hline 0 & 5 & 12.8 & 7 & 17.1 & 12 & 15.0 & \\
\hline $1-4$ & 18 & 46.2 & 20 & 48.8 & 38 & 47.5 & \\
\hline$>4$ & 16 & 41.0 & 14 & 34.1 & 30 & 37.5 & \\
\hline \multicolumn{8}{|l|}{ Visible plaque index } \\
\hline$\leq 20 \%$ & 18 & 46.2 & 24 & 28.5 & 42 & 52.5 & $0.366^{\circ}$ \\
\hline$>20 \%$ & 21 & 53.8 & 17 & 41.5 & 38 & 47.5 & \\
\hline \multicolumn{8}{|l|}{ Gingival blood index } \\
\hline$\leq 10 \%$ & 33 & 84.6 & 35 & 85.4 & 68 & 85.0 & $0.224^{b}$ \\
\hline$>10 \%$ & 6 & 15.4 & 6 & 14.6 & 12 & 15.0 & \\
\hline \multicolumn{8}{|c|}{$N^{\circ}$ active non-cavitated lesions } \\
\hline$\leq 5$ & 24 & 61.5 & 27 & 65.9 & 51 & 63.8 & $0.673^{\circ}$ \\
\hline$>5$ & 15 & 38.5 & 14 & 34.1 & 29 & 36.2 & \\
\hline Total & 39 & 100 & 41 & 100 & 80 & 100 & \\
\hline
\end{tabular}

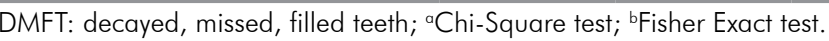

sugar intake. There is a need to develop feasible standardized methods to assess free sugar intake to facilitate surveillance across populations. ${ }^{22}$

Of the 47 lesions that remained active after treatment, 33 were in permanent teeth, and most of them were on smooth surfaces. It is known that deciduous enamel has a higher number of organic components than the permanent enamel. The prism diameter measurement in deciduous teeth is also smaller, resulting in greater porosity of the enamel and increasing its fragility ${ }^{23}$. The difference in chemical behavior of the enamel can probably modify the structural characteristics and rate of caries lesion progression. In addition, lesions on permanent teeth may be newer lesions with less time in the oral cavity, because they are in newly erupted teeth. These lesions are the ones that are usually active during the eruption period and would later become inactive anyway. Backer Dirks ${ }^{24}$ have already reported that many of the carious lesions on the buccal surfaces that developed soon after eruption were intact or unchanged after 7 years.

There was no additional effect of associating the $1.23 \%$ APF gel with fluoride dentifrice on the arrest of non-cavitated carious lesions. Moreover, a placebo effect may also have occurred in the NFG group. Unfortunately, no conclusion was reached to determine the ideal TGA frequency necessary. The mean number of weeks until the total arrest was five in both groups. The 8-week TGA protocol was based on previous studies. ${ }^{10,11}$

Only two double-blind randomized controlled school-based trials in permanent dentition with schoolchildren aged 7 to 12 years have been published. The studies found no additional benefits after treating 
Table 2. Association between caries lesion control and explanatory variables (simple and multiple regression models).

\begin{tabular}{|c|c|c|c|c|}
\hline \multirow{2}{*}{ Variable } & \multirow{2}{*}{$\begin{array}{l}\text { Simple } \\
\text { p-value }\end{array}$} & \multicolumn{3}{|c|}{ Multiple } \\
\hline & & OR & $\mathrm{p}$-value & $95 \% \mathrm{Cl}$ \\
\hline \multicolumn{5}{|l|}{ Group } \\
\hline Fluoride gel $(n=39)$ & & 1 & & \\
\hline Non-fluoride gel $(n=41)$ & 0.33 & 0.68 & 0.33 & $(0.32-1.48)$ \\
\hline \multicolumn{5}{|l|}{ Mother's educational level } \\
\hline Elementary school $(n=20)$ & & 1 & & \\
\hline High school $(n=46)$ & & 0.59 & 0.29 & $(0.22-1.58)$ \\
\hline Higher education $(n=14)$ & 0.36 & 0.88 & 0.88 & $(0.17-4.65)$ \\
\hline \multicolumn{5}{|l|}{ Dentition } \\
\hline Deciduous $(n=21)$ & & 1 & & \\
\hline Mixed $(n=41)$ & & 1.9 & 0.27 & $(0.60-6.12)$ \\
\hline Permanent $(n=18)$ & 0.42 & 1.2 & 0.76 & $(0.30-4.98)$ \\
\hline \multicolumn{5}{|l|}{ DMFT Baseline } \\
\hline $0(n=12)$ & & 1 & & \\
\hline $1-4(n=38)$ & & 1.1 & 0.78 & $(0.33-4.23)$ \\
\hline$>4(n=30)$ & 0.59 & 2.0 & 0.30 & $(0.53-7.65)$ \\
\hline \multicolumn{5}{|l|}{ Visible plaque index baseline } \\
\hline$\leq 20 \%(n=42)$ & & 1 & & \\
\hline$>20 \%(n=38)$ & 1.0 & 0.91 & 0.86 & $(0.35-2.39)$ \\
\hline \multicolumn{5}{|l|}{ Gingival blood index baseline } \\
\hline$\leq 10 \%(n=68)$ & & 1 & & \\
\hline$>10 \%(n=12)$ & 1.0 & 0.96 & 0.95 & $(0.33-2.81)$ \\
\hline \multicolumn{5}{|l|}{ Toothbrushing frequency } \\
\hline $1 \mathrm{x} /$ day $(\mathrm{n}=11)$ & & 1 & & \\
\hline $2 x /$ day $(n=38)$ & & 0.50 & 0.13 & $(0.20-1.22)$ \\
\hline$\geq 3 x /$ day $(n=31)$ & 0.24 & 0.31 & 0.04 & $(0.10-0.95)$ \\
\hline \multicolumn{5}{|c|}{ Frequency of sugar intake baseline } \\
\hline $0-5 x /$ day $(n=52)$ & & 1 & & \\
\hline$>5 x /$ day $(n=28)$ & 0.78 & 0.75 & 0.58 & $(0.27-2.06)$ \\
\hline
\end{tabular}

$\mathrm{Cl}$ : confidence interval; DMFT: decayed, missed, filled teeth.

incipient carious lesions with fluoride gel. ${ }^{10,11}$ Ferreira et al. ${ }^{10}$ treated white spots on the buccal surfaces of permanent upper incisors once a week for 8 weeks. The gel was applied to all the teeth with a cotton swab. The children were supervised while brushing with non-fluoride dentifrice, and they lived in a non-fluoridated water area. The results did not differ significantly between the groups. The study suggests that weekly supervised toothbrushing was able to arrest enamel white spots.
Bonow et al. ${ }^{11}$ treated active incipient non-cavitated carious lesions on the buccal surfaces of the upper permanent incisors and/or lower permanent first molars. The gel was applied weekly for 8 weeks only on the lesions using cotton swabs. Children were exposed to fluoridated water and dentifrice. This trial demonstrates the equivalence of the treatments. Professional dental plaque removal may also account for the resulting equivalence of the treatments. The necessity of topical applications 
of high fluoride concentration for caries control, and the appropriate intervals for gel application would appear to be unimportant. There is a very clear need for studies on the best fluoride regimen to assist in the remineralization of early carious lesions. ${ }^{9}$

No clinical difference was observed in the enamel of inactive non-cavitated lesions when APF or placebo gel was applied. However, in vitro and in situ studies ${ }^{8,9}$ have shown the efficacy of APF gel in the control of non-cavitated carious lesions, even when used in association with fluoride dentifrice. The results were not clinically detectable, but enamel presenting a higher amount of fluoride and higher mineral content was observed, probably leading to a higher resistance to future acid challenges. Based on these results, longitudinal studies should be performed to follow the enrolled and treated children in the present study to verify whether a difference in the new carious lesions could be observed.

It is important to note that this study was performed in an extremely controlled environment, under an intensive preventive treatment protocol, making its external validity and applicability one of the limitations of the study.

\section{Conclusion}

Based on the findings of this controlled study, there is no additional effect of the association of $1.23 \%$ APF gel with oral hygiene (fluoride dentifrice) and dietary instructions on the arrest of active non-cavitated lesions in permanent, mixed, and primary dentitions in children between 3 and 12 years of age. Therefore, the null hypothesis was not rejected. We also confirmed the importance of the frequency of toothbrushing and plaque reduction in the control of caries activity.

\section{Acknowledgements}

We thank the National Coordination of Post-Graduate Education (Capes), Pro-Rectory of Research of the Federal University of Rio Grande do Sul (Propesq), DFL Brazil, and Colgate/Palmolive, Brazil for their support.

\section{References}

1. Gibson S, Williams S. Dental caries in pre-school children: associations with social class, toothbrushing habit and consumption of sugars and sugar-containing foods. Caries Res. 1999;33(2):101-13. https://doi.org/10.1159/000016503

2. Gillcrist JA, Brumley DE, Blackford JU. Community socioeconomic status and children's dental health. J Am Dent Assoc. 2001 Feb;132(2):216-22. https://doi.org/10.14219/jada.archive.2001.0158

3. Ismail Al, Brodeur JM, Gagnon P, Payette M, Picard D, Hamalian T, et al. Prevalence of non-cavitated and cavitated carious lesions in a random sample of 7-9-year-old schoolchildren in Montreal, Quebec. Community Dent Oral Epidemiol. 1992 Oct;20(5):250-5. https://doi.org/10.1111/j.1600-0528.1992.tb01693.x

4. Machiulskiene V, Nyvad B, Baelum V. Prevalence and severity of dental caries in 12-year-old children in Kaunas, Lithuania 1995. Caries Res. 1998;32(3):175-80. https://doi.org/10.1159/000016450

5. Kassawara AB, Tagliaferro EP, Cortelazzi KL, Ambrosano GM, Assaf AV, Meneghim MC, et al. Epidemiological assessment of predictors of caries increment in 7-10-year-olds: a 2-year cohort study. J Appl Oral Sci. 2010 Mar-Apr;18(2):116-20. https://doi.org/10.1590/S1678-77572010000200004

6. Nyvad B, Fejerskov $\mathrm{O}$. Assessing the stage of caries lesion activity on the basis of clinical and microbiological examination. Community Dent Oral Epidemiol. 1997 Feb;25(1):69-75. https://doi.org/10.1111/j.1600-0528.1997.tb00901.x

7. Clarkson B, Fejerskov $O$, Silverstone LN. Rational use of fluorides in caries prevention and treatment. In: Clarkson B, Fejerskov $O$, Silverstone LN, editors. Fluoride in dentistry. Copenhagen: Munksgaard; 1988. p. 276-388.

8. Delbem AC, Brighenti FL, Vieira AE, Cury JA. In vitro comparison of the cariostatic effect between topical application of fluoride gels and fluoride toothpaste. J Appl Oral Sci. 2004 Jun;12(2):121-6. https://doi.org/10.1590/S1678-77572004000200008

9. Jardim JJ, Pagot MA, Maltz M. Artificial enamel dental caries treated with different topical fluoride regimes: an in situ study. J Dent. 2008 Jun;36(6):396-401. https://doi.org/10.1016/i.jdent.2008.02.010

10. Ferreira MA, Latorre MR, Rodrigues CS, Lima KC. Effect of regular fluoride gel application on incipient carious lesions. Oral Health Prev Dent. 2005;3(3):141-9. 
11. Bonow ML, Azevedo MS, Goettems ML, Rodrigues CR. Efficacy of 1.23\% APF gel applications on incipient carious lesions: a double-blind randomized clinical trial. Braz Oral Res. 2013 May-Jun;27(3):279-85. https://doi.org/10.1590/S1806-83242013000300007

12. Bader JD, Shugars DA, Bonito AJ. A systematic review of selected caries prevention and management methods. Community Dent Oral Epidemiol. 2001 Dec;29(6):399-411. https://doi.org/10.1034/i.1600-0528.2001.290601.x

13. Ainamo J, Bay I. Problems and proposals for recording gingivitis and plaque. Int Dent J. 1975 Dec;25(4):229-35.

14. Ismail Al, Sohn W, Tellez M, Amaya A, Sen A, Hasson H, et al. The International Caries Detection and Assessment System (ICDAS): an integrated system for measuring dental caries. Community Dent Oral Epidemiol. 2007 Jun;35(3):170-8. https://doi.org/10.1111/j.1600-0528.2007.00347.x

15. Nyvad B, Machiulskiene V, Baelum V. Reliability of a new caries diagnostic system differentiating between active and inactive caries lesions. Caries Res. 1999 Jul-Aug;33(4):252-60. https://doi.org/10.1159/000016526

16. Oliveira RS, Zenkner JE, Maltz M, Rodrigues JA. Effectiveness of a standardized treatment protocol for children with active non-cavitated occlusal lesions on erupting permanent molars. Int J Paediatr Dent. 2015 Nov;25(6):393-8. https://doi.org/10.1111/ipd.12141

17. Kumar S, Tadakamadla J, Johnson NW. Effect of toothbrushing frequency on incidence and increment of dental caries: a systematic review and meta-analysis. J Dent Res. 2016 Oct;95(11):1230-6. https://doi.org/10.1177/0022034516655315

18. Treasure E, Kelly M, Nuttall N, Nunn J, Bradnock G, White D. Factors associated with oral health: a multivariate analysis of results from the 1998 Adult Dental Health survey. Br Dent J. 2001 Jan;190(2):60-8. https://doi.org/10.1038/si.bdj.4800886

19. Holmen L, Thylstrup A, Artun J. Surface changes during the arrest of active enamel carious lesions in vivo: a scanning electron microscope study. Acta Odontol Scand. 1987 Dec;45(6):383-90. https://doi.org/10.3109/00016358709096362

20. Paes Leme AF, Tabchoury CP, Zero DT, Cury JA. Effect of fluoridated dentifrice and acidulated phosphate fluoride application on early artificial carious lesions. Am J Dent. 2003 Apr;16(2):91-5.

21. Urteaga Ribbeck C, Fernandes ACP, Samur EA. [Comparison of results from two dietary interview methods]. Arch Latinoam Nutr. 2003 Jun;53(2):172-7. Spanish.

22. Moynihan PJ, Kelly SA. Effect on caries of restricting sugars intake: systematic review to inform WHO guidelines. J Dent Res. 2014 Jan;93(1):8-18. https://doi.org/10.1177/0022034513508954

23. Silness J, Hegdahl T, Gustavsen F. Area of the organic-inorganic interface of dental enamel. Acta Odontol Scand. 1973;31 (2):123-9. https://doi.org/10.3109/00016357309004618

24. Backer Dirks OB. Posteruptive changes in dental enamel. J Dent Res. 1966;45(3):503-11. https://doi.org/10.1177/00220345660450031101 